PSYCHOMETRIKA-VOL. 50, NO. 4, 479-494

DECEMBER 1985

\title{
EXTERNAL ANALYSIS WITH THREE-MODE PRINCIPAL COMPONENT MODELS
}

\author{
Willem A. VAN DER KLOOT \\ LEIDEN UNIVERSITY \\ Pieter M. KroOnenberg \\ DEPARTMENT OF EDUCATION \\ LEIDEN UNIVERSITY
}

\begin{abstract}
Through external analysis of two-mode data one attempts to map the elements of one mode (e.g., attributes) as vectors in a fixed space of the elements of the other mode (e.g., stimuli). This type of analysis is extended to three-mode data, for instance, when the ratings are made by more individuals. It is described how alternating least squares algorithms for three-mode principal component analysis (PCA) are adapted to enable external analysis, and it is demonstrated that these techniques are useful for exploring differences in the individuals' mappings of the attribute vectors in the fixed stimulus space. Conditions are described under which individual differences may be ignored. External three-mode PCA is illustrated with data from a person perception experiment, designed after two studies by Rosenberg and his associates whose results were used as external information.
\end{abstract}

Key words: multidimensional scaling; individual differences, three-mode factor analysis, person perception.

A classical problem in multidimensional scaling (MDS) concerns the interpretation of the derived stimulus configurations. This problem is usually handled by procuring separate ratings of the stimuli on a set of attributes, and mapping those attributes as vectors into the stimulus space (for an early application see Rosenberg, Nelson \& Vivekanantan, 1968). This approach may be called external analysis (cf. Carroll, 1972, p. 114). To achieve the same goal procedures to relate external variables or éléments supplémentaires to solutions of correspondence analysis have been developed by Benzécri (1976, p. $36 \mathrm{ff}$; Benzécri \& Benzécri, 1980, p. $5 \mathrm{ff}$.) and have been extended to two-way versions of multiway tables by Cazes (1982). One kind of extension of such external procedures is to use other models than the vector model (e.g., unfolding models) and represent the attributes not as vectors but as points in the given configurations. This option is implemented in the well-known computer program PREFMAP (Carroll, 1972).

A second kind of extension is to represent linear combinations of the attributes as vectors in the stimulus space instead of mapping each attribute separately. This can be done, for instance, by means of canonical correlation analysis (Schiffman, Reynolds, \& Young, 1981, p. 282 ff.) or through redundancy analysis (Rao, 1964; van den Wollenberg, 1977).

A special situation arises when the data matrix is three-way/three-mode, for instance, when the stimuli are rated on each attribute by a number of judges. This case is usually

We gratefully acknowledge the assistance of Piet Brouwer in implementing the external analysis options in the TUCKALS programs.

Requests for reprints should be sent to Willem A. van der Kloot, Department of Psychology, Hooigracht 15, 2312 KM Leiden, THE NETHERLANDS.

Correspondence regarding the TUCKALS programs should be addressed to Pieter M. Kroonenberg, Department of Education, Postbus 9507, 2300 RA Leiden, THE NETHERLANDS. 
handled by aggregating the ratings over the judges in order to obtain one attribute score for each stimulus. Thus, the judges are treated as replications of each other, and the problem is reduced to finding a representation of the mean (or median) attribute scores in the stimulus space. Since individual differences between judges are neglected in this procedure, averaging seems only adequate if the judges can be assumed to form a more or less homogeneous group. Homogeneity in this context means that there are no systematic individual differences between judges or that such differences are small or only of a special kind (as will be discussed below).

If we want to represent a judge's ratings of the stimuli on an attribute as a vector in the stimuli space, then individual differences between judges will consist of differences in direction and/or differences in length of the attribute vectors. Differences in direction indicate that the judges involved interpret the stimulus space and/or the attribute in different ways, because the projections of the (fixed) stimuli on the attribute vectors are different. When the attribute vectors differ only in length, the projections of the stimuli are the same for all judges. Therefore, aggregating the ratings of judges is only useful if their attribute vectors differ in length and have at most minor fluctuations in direction. In such a case, the direction of the "mean" vector will be representive for the individual vectors. However, when the attribute vectors of judges differ considerably in direction, aggregation makes less sense, because the mean vector will not be representive for the ratings of at least a number of judges. Therefore, we prefer a method of external analysis that does justice to possible differences in directions, and indicates whether or not a group of judges may be regarded as homogeneous in this respect.

Instead of fitting the attribute vectors of each person separately we propose to use a third kind of extension, namely external three-mode principal component analysis (PCA) (Kroonenberg, 1983; Kroonenberg \& de Leeuw, 1980; Tucker, 1966). ${ }^{1}$ This method, which was first presented by Kroonenberg, van der Kloot and Brouwer (1983), is preferred because it does not only yield a "mean" or "common" representation of the attributes, but also indicates whether and to what extent individual judges differ with respect to their "use" of this common representation. Moreover, possible individual differences can be decomposed into a (small) number of components, each of which corresponds to a particular pattern of relations between the stimuli and the attributes.

In the present paper we will discuss external analysis with the three-mode PCA programs (TUCKALS2 and TUCKALS3) developed by Kroonenberg and de Leeuw (1980). Similar external analysis options based on the same algorithmic principles are included in other programs for three-way data: ALSCOMP (Sands \& Young, 1980), ALSCAL (Young \& Lewyckyj, 1979), INDSCAL-CANDECOMP (Carroll \& Chang, 1970), MULTISCALE (Ramsay, 1982), and PARAFAC (Harshman \& Lundy, 1984a). It should be noted, however, that these programs are based on simpler models, and that, to our knowledge, there exist no formally published applications of these methods.

We will start with a discussion how individual differences are accounted for in the models underlying TUCKALS2 and TUCKALS3, and we will present some technical aspects of adapting the TUCKALS algorithms for several forms of external analysis. Finally, we will describe applications of such analyses and compare their results with those of an "unconstrained" analysis.

\section{TUCKALS2}

The model underlying the TUCKAL2 algorithm is

$$
Z_{k} \simeq G C_{k} H^{\prime}, \quad(k=1, \ldots, n)
$$

where $Z_{k}$ (e.g., the ratings of subject $k$ ) is the $k$-th "slice" of the $(\ell \times m \times n)$ three-way data matrix $Z$, and $\simeq$ indicates that the right-hand side is a least-squares approximation of the 
left-hand side. The orthonormal $(\ell \times s)$ matrix $G$ and the orthonormal $(m \times t)$ matrix $H$ represent the loadings of the elements of the first two modes (e.g., stimuli and attributes), and $C_{k}$ is the $k$-th slice of the $(s \times t \times n)$ core matrix $C . C_{k}$, which is associated with element $k$ of the third mode, describes the way in which $G$ and $H$ are related for subject $k$. For instance, $c_{p q}^{k}$ of $C_{k}$ indicates the strength and sign of the relationship between the $p$-th component of the first mode and the $q$-th component of the second mode for subject $k$.

When $G$ and $H$ are columnwise orthonormal, $\left(c_{p q}^{k}\right)^{2}$ is the amount of explained variation by the combination of components $g_{p}$ and $h_{q}$ for subject $k$. Within this model, external analysis is possible by fixing either $G$ or $H$, or both. If we let $G$ equal a set of fixed stimulus loadings, the objective of an external analysis is to obtain estimates of all $C_{k}$ and of $H$, the loadings of, say, the attributes on a specified number of components. $H$ describes the best fitting attribute space for all subjects simultaneously, and $C_{k}$ describes the way in which a particular subject $k$ links the components of the attribute space to the components of the dimensions of the fixed stimulus space. The estimates of $H$ and $C_{k}$ can be used to plot the directions of the attributes and their components for subject $k$ in the common stimulus space.

Since $H$ is the common matrix of attribute loadings, individual differences between the subjects show up as differences between the $C_{k}$ matrices. Differences between these matrices correspond to individual differences among the judges that involve both the directions and/or the lengths of their attribute vectors. In the remainder of this section we will discuss several types of such individual differences.

Differences in length only. It can be shown that two judges, $k$ and $k$, have attribute vectors that differ only in length if and only if

$$
C_{k} H^{\prime}=C_{k^{\prime}} H^{\prime} \Lambda_{k k^{\prime}} \quad\left(k, k^{\prime}=1, \ldots, n\right)
$$

with $\Lambda_{k k^{\prime}}$ being a diagonal matrix of dimensions $m \times m$, which may be different for each combination of $k$ and $k^{\prime}$. When the elements of $\Lambda_{k k^{\prime}}$ differ among each other, the differences in vector length vary from attribute to attribute. Some attribute vectors may be larger for subject $k$ and some may be larger for subject $k^{\prime}$. Also, the extent to which a vector of subject $k$ is larger than the corresponding vector of subject $k^{\prime}$ varies from attribute to attribute. A special case occurs when all the elements of $\Lambda_{k k^{\prime}}$ are the same. In that case (2) reduces to

$$
C_{k} H^{\prime}=\lambda_{k k^{\prime}} C_{k^{\prime}} H^{\prime} \quad \text { or } \quad C_{k}=\lambda_{k k^{\prime}} C_{k^{\prime}},
$$

which means that the lengths of all vectors of subject $k$ are proportional by a factor $\lambda_{k k^{\prime}}$ to the length of those of subject $k^{\prime}$. Figure 1 shows an example of this type of differences.

Differences in direction. Three special cases will be considered here.

1. The simplest case arises when all $C_{k}$ matrices are diagonal, so that the judges differ only with respect to the diagonal entries of their $C_{k}$. This case is a variant of the CANDECOMP/PARAFAC model (Carroll \& Chang, 1970; Harshman, 1970; Harshman \& Lundy, 1984a) with orthogonal $G$ and $H$. A diagonal $C_{k}$ indicates that the dimensions of the stimuli and those of the attributes coincide, and that the subjects differ only with respect to the weights they assign to these common dimensions. Figure 1 displays the attribute components and the separate attribute vectors in the stimulus space for three diagonal matrices $C_{k}$. It is clear that the attribute components have the same orientation, though this need not be the case for the attribute vectors themselves. The configurations of the separate vectors of $C_{k} H^{\prime}$ and $C_{k^{\prime}} H^{\prime}$ appear to be substantially different. When $C_{k}$ is proportional to $C_{k^{\prime}}$ the vectors of subject $k$ and $k^{\prime}$ will be oriented in the same manner; this is, however, only a sufficient condition and not a necessary one. 

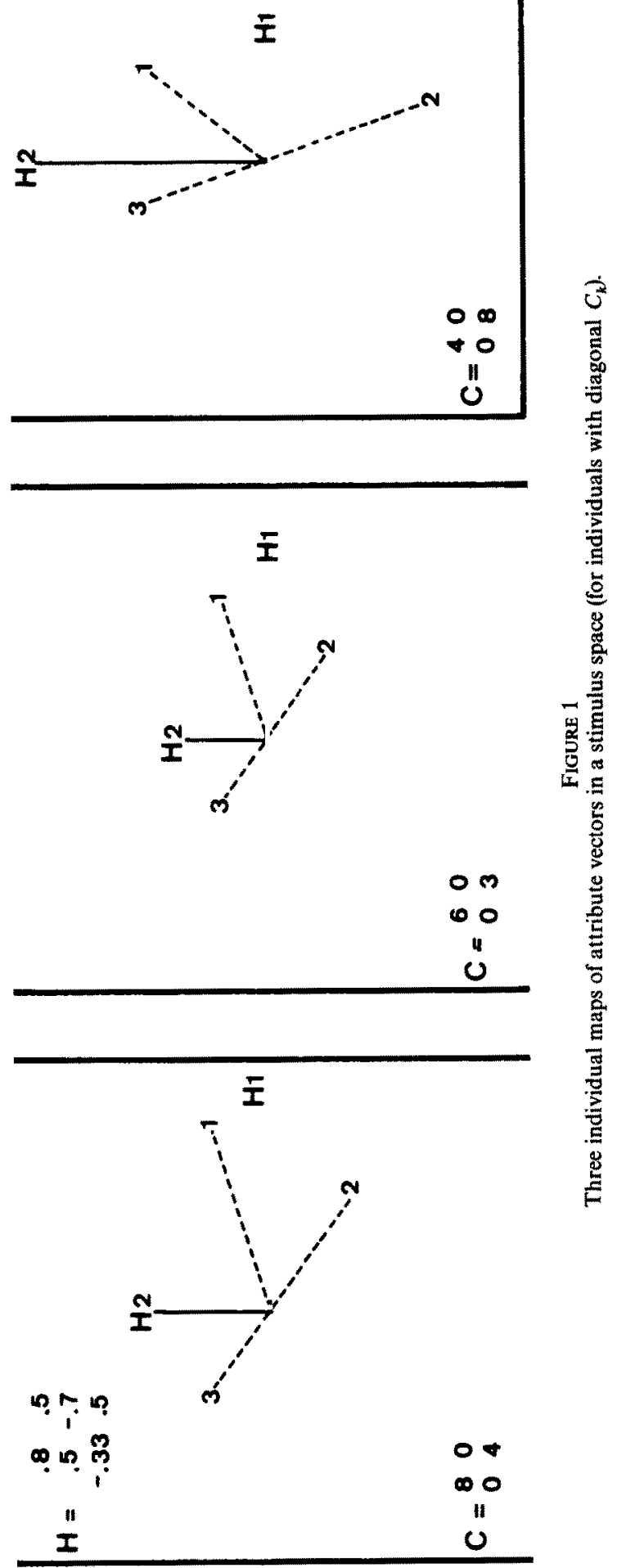
2. Another type of individual differences occurs if each $C_{k}$ matrix is equal to a product of the form

$$
C_{k}=D_{k}^{1 / 2} R D_{k}^{1 / 2},
$$

where $D_{k}$ is a diagonal matrix containing the non-negative weights given by subject $k$ to the dimensions of the stimulus and attribute spaces, and $R$ is a symmetric matrix that indicates how the attribute components are oriented with respect to the stimulus space (and vice versa). This model differs from the former one in that the attribute components may be nonorthogonal to each other (see Tucker, 1972, p. 7). Different $D_{k}$ matrices correspond to different weights assigned to the attribute dimensions. This is graphically represented by a different stretching or shrinking of those axes (see Figure 2). This model is a variant of the so-called PARAFAC2 model presented by Harshman (1972) and Harshman and Lundy (1984a).

3. The most general case of individual differences occurs when all $C_{k}$ matrices are in principle unrestricted with respect to their form or to the elements they contain. In this zase it is possible that the $C_{k}$ matrix of subject $k$ is a combination of a few basic types of $C$ matrices. This possibility underlies the model fitted by TUCKALS3, which we will discuss in the next paragraph.

\section{TUCKALS3}

The model that underlies the TUCKALS3 algorithm is

$$
Z \simeq G C\left(E^{\prime} \otimes H^{\prime}\right)
$$

where $Z$ is the $(\ell \times m \times n)$ three-way data matrix rearranged as a row supervector consisting of $n$ frontal slices of order $\ell \times m$; this will be denoted by $Z \in \mathbb{R}^{\ell \times m n}$. $G$ and $H$ are the orthonormal $(\ell \times s)$ and $(m \times t)$ matrices containing the loadings of the stimuli and the attributes. $\otimes$ is the Kronecker product. $E$ is the orthonormal $(n \times u)$ matrix of component loadings for the elements of the third mode, for example the subjects, and $C=$ $\left\{c_{p q r}\right\}$ is the $(s \times t \times u)$ core matrix that displays the relationships between the components of the three modes rearranged as a row supervector consisting of $u(s \times t)$ matrices, that is, $C \in \mathbb{R}^{s \times t u}$. The $c_{p q r}^{2}$ represents the amount of explained variation of the combination of the $p$-th component of the first mode, the $q$-th component of the second mode, and the $r$-th component of the third mode.

Another way of writing (5) is

$$
Z_{k} \simeq G\left(e_{k 1} C_{1}+e_{k 2} C_{2}+\cdots+e_{k u} C_{u}\right) H^{\prime} \quad(k=1, \ldots, n)
$$

What this model amounts to is that to each component of $E$ corresponds a particular slice of $C$ (i.e., $C_{1}, C_{2}, \ldots, C_{u}$ ) that describes the relationships between the stimulus and attribute components as "seen" by a subject who has zero loadings on the other components of $E$. If $E$ has only one component, that is, when the subject space is onedimensional, (6) is reduced to

$$
Z_{k} \simeq G\left(e_{k} C\right) H^{\prime}
$$

In this special case all $C_{k}$ matrices are proportional to each other, which implies that for all subjects the same attributes have the same direction.

\section{Technical Aspects}

In the TUCKALS2 and TUCKALS3 algorithms the parameters are estimated by minimizing least squares loss functions in which the component matrices are restricted to be orthonormal. These orthonormality restrictions may be made without loss of gener- 

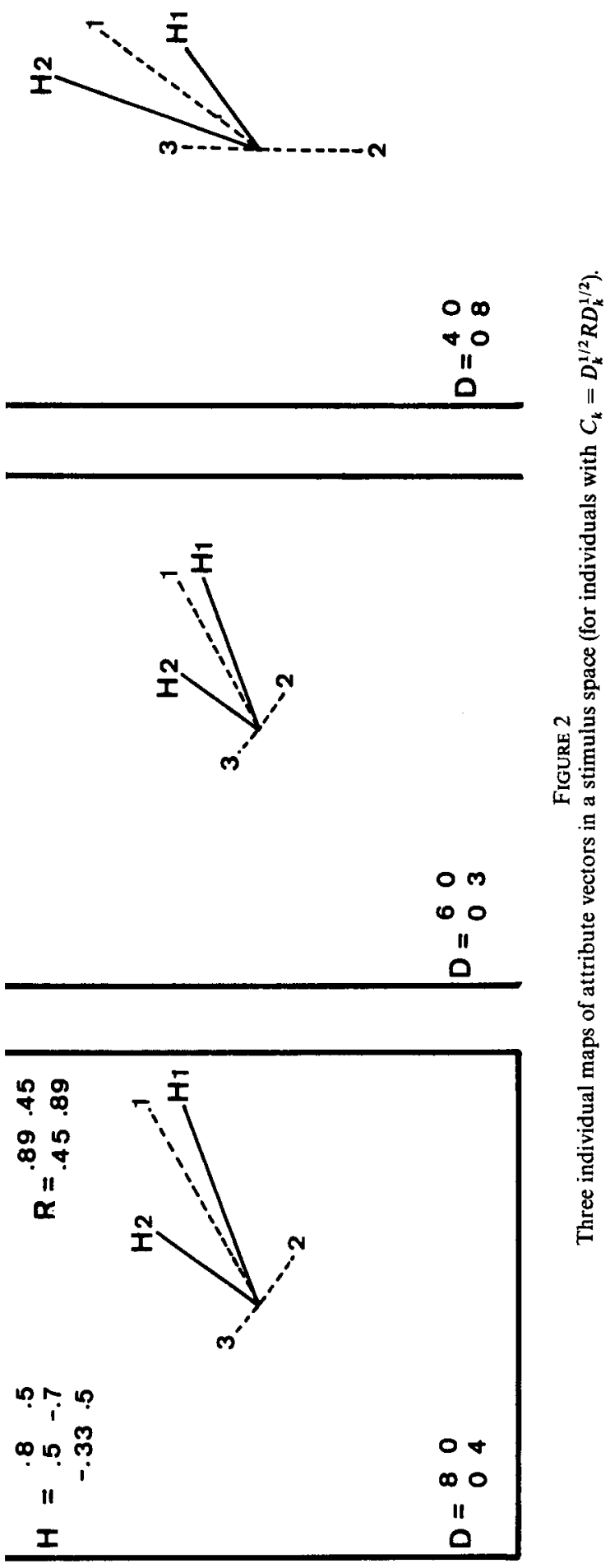
ality; they are imposed to identify the minimization equations and for computational efficiency. They may be relaxed after the parameter values have been determined.

TUCKALS2. The loss function minimized in TUCKALS2 has the form

$$
f(G, H, C)=\sum_{k=1}^{n}\left\|Z_{k}-G C_{k} H^{\prime}\right\|^{2} \quad \text { with } \quad G^{\prime} G=I_{s} \quad \text { and } \quad H^{\prime} H=I_{t},
$$

where $\|\cdot\|$ indicates the Euclidean norm, and $I_{a}$ is the $a \times a$ identity matrix. The minimization of $f$ over $G, H$ and $C$ may be written as (see Kroonenberg \& de Leeuw, 1980, p. 71)

$$
\begin{aligned}
\min _{G, H, C} f(G, H, C) & =\min _{G, H}\left\{\min _{c} f(G, H, C)\right\}, \\
& =\min _{G, H}\left\{\min _{c} \sum_{k=1}^{n}\left\|Z_{k}-G C_{k} H^{\prime}\right\|^{2}\right\}, \\
& =\min _{G, H} \sum_{k=1}^{n}\left\|Z_{k}-G G^{\prime} Z_{k} H H^{\prime}\right\|^{2}, \\
& =\min _{G, H}\left\{t r \sum_{k=1}^{n} Z_{k}^{\prime} Z_{k}-\operatorname{tr} H^{\prime}\left(\sum_{k=1}^{n} Z_{k}^{\prime} G G^{\prime} Z_{k}\right) H\right\} ; \\
& =\operatorname{tr} \sum_{k=1}^{n} Z_{k}^{\prime} Z_{k}-\max _{G, H} \operatorname{tr} H^{\prime} Q H
\end{aligned}
$$

with $Q=\sum_{k=1}^{n} Z_{k}^{\prime} G G^{\prime} Z_{k}$, and $G$ and $H$ orthonormal. Alternatively, (8) may be written as

$$
\begin{gathered}
\min _{G, H}\left\{\operatorname{tr} \sum_{k=1}^{n} Z_{k}^{\prime} Z_{k}-\operatorname{tr} G^{\prime}\left(\sum_{k=1}^{n} Z_{k} H H^{\prime} Z_{k}^{\prime}\right) G\right\} \\
=\operatorname{tr} \sum_{k=1}^{n} Z_{k}^{\prime} Z_{k}-\max _{G} \operatorname{tr} G^{\prime} P G
\end{gathered}
$$

with $P=\sum_{k=1}^{n} Z_{k} H H^{\prime} Z_{k}^{\prime}$.

When $G$ is fixed $Q$ is fixed as well, and the maximization in (9) becomes a standard eigenvalue-eigenvector problem. Its solution $H$ is the eigenvector matrix corresponding to the $t$ largest eigenvalues of $Q$. Similarly, when $H$ is fixed $P$ is fixed as well, and thus the maximization in (11) has as its solution the eigenvector matrix $G$ corresponding to the $s$ largest eigenvalues of $P$. The TUCKALS2 algorithm alternates between the maximizations in (9) and (11) until convergence is reached. After convergence $C=\left(C_{1}, C_{2}, \ldots, C_{n}\right)$ is computed as $C_{k}=G^{\prime} Z_{k} H(k=1, \ldots, n)$, which follows from the minimization of the loss function (8) over $C$ for known $G$ and $H$ (for further details see Kroonenberg, 1983, chap. 4, or Kroonenberg \& de Leeuw, 1980).

If in an external analysis $G$ is fixed, only (9) needs to be solved as it is not necessary to solve (11). Similarly, when $H$ is fixed only (11) needs to be solved, and when both $G$ and $H$ are fixed only $C$ needs to be computed. In these cases it is not necessary to use an alternating least squares procedure such as TUCKALS2. The TUCKALS2 program may, however, be fruitfully used for external analysis when one wants to compare the results with those of an analysis without fixed components (an unconstrained analysis; see also below).

TUCKALS3. The loss function minimized in TUCKALS3 has the form

$$
h(G, H, E, C)=\|Z-\hat{Z}\|^{2}
$$


with

$$
\hat{Z}=G C\left(E^{\prime} \otimes H^{\prime}\right) \quad \text { and } \quad Z, \hat{Z} \in \mathbb{R}^{\ell \times m n}, \quad C \in \mathbb{R}^{s \times t u} .
$$

As indicated in detail in Kroonenberg and de Leeuw (1980) optimal values for $G=\left\{g_{i p}\right\}$, $H=\left\{h_{j q}\right\}$ and $E=\left\{e_{k r}\right\}$ may be found by alternating between the following three steps of the TUCKALS3 algorithm.

Step 1:

Maximize tr $G^{\prime} P G$ over $G$

with

$$
P=\{Z(E \otimes H)\}\{Z(E \otimes H)\}^{\prime} \quad \text { and } \quad Z \in \mathbb{R}^{\ell \times m n} \text {. }
$$

Step 2:

Maximize tr $H^{\prime} Q H$ over $H$

with

$$
Q=\{Z(G \otimes E)\}\{Z(G \otimes E)\}^{\prime} \text { and } Z \in \mathbb{R}^{m \times n \ell} \text {. }
$$

Step 3:

Maximize tr $E^{\prime} R E$ over $E$

with

$$
R=\{Z(H \otimes G)\}\{Z(H \otimes G)\}^{\prime} \text { and } Z \in \mathbb{R}^{n \times \ell m} \text {. }
$$

Comparison with steps (9) and (11) shows that in TUCKALS3 simultaneously three different eigenvalue-eigenvector problems have to be solved. $C$ may be found from

$$
C=G^{\prime} Z(E \otimes H),
$$

after convergence of the algorithm for $G, H$ and $E$.

External analysis with TUCKALS3 can now have three basic forms. First, only one component matrix, say $G$, is fixed. This means that (14) may be skipped and that the other two component matrices can be found by solving (15) and (16) iteratively, and computing $C$ by (17) after convergence. Secondly, two of the component matrices, say $G$ and $H$, may be fixed, in which case only (16) and (17) need to be solved. Finally, when all three component matrices are fixed, only $C$ needs to be computed via (17). Thus only in the case that just one component matrix is held fixed there is any need to use an alternating least squares algorithm. In the other two cases it may still be worthwhile to use the TUCKALS3 program in order to compare a constrained solution (i.e., a solution with fixed components) with a solution without fixed components.

\section{Comparing solutions}

As was shown above, a sufficient condition for aggregating stimulus ratings across subjects is met when the individual $C_{k}$ are proportional to each other. Whether this is the case can be determined from a TUCKALS3 analysis: If the subject mode is onedimensional, all $C_{k}$ are proportional. The opposite case consists of all subjects having different $C_{k}$ matrices that cannot be decomposed into a small number of underlying dimensions. If this is true, the fit of an (external) TUCKALS2 solution should be (much) greater than the fit of an (external) TUCKALS3 solution in one (or a few) dimensions. Therefore, in practical applications of external three-mode PCA one would want to compare the fit measures of TUCKALS2 and TUCKALS3 solutions.

The fit of a solution is defined to be tr $\hat{Z}^{\prime} \hat{Z}$, that is the sum of the squared fitted data. Furthermore, the fit is equal to the sum of the squared elements of the core matrix, and is also equal to the sums of the component weights in each mode. The weights of the components of the first, second, and third mode are the eigenvalues associated with the eigenvectors $G, H$, and $E$ of $P, Q$, and $R$ as defined in (14), (15), and (16) respectively (for further details see Kroonenberg, 1983, chap. 6).

Even when the main goal of a study is external analysis, it is sometimes worthwhile to perform one or more internal or unconstrained analyses as well. Fit and dimensionality of an unconstrained solution indicate whether the fit of an external solution can be substantially improved by "freeing" the fixed configuration. In this context one could con- 
ceivably use the descriptive fit measures presented by Bentler and Bonett (1980) and Bonett and Bentler (1983), but we will not pursue this topic here.

Centering for external analysis

A special problem in three-mode PCA (here discussed in terms of TUCKALS2) is the centering of the input data (see Harshman \& Lundy, 1984b; Kroonenberg, 1983, chap. 6; Kruskal, 1981, 1983a), because the way in which $Z$ is centered has consequences for the centroids of $G$ and/or $H$. For instance, centering each column of $Z_{k}$ yields a $G$ matrix that is columnwise centered, whereas centering each row of $Z_{k}$ yields an $H$ with zero column sums. Double centering of each $Z_{k}$ causes the centroids of both $G$ and $H$ to be in the origins of their respective spaces.

As a general principle for external analysis, it seems advisable to center the $Z_{k}$ in such manner that an unconstrained analysis would yield a $G$ (and/or $H$ ) that is centered in the same manner as the fixed $G$ (and/or $H$ ) in the external analysis. This ensures that if the fixed space happens to be identical to the solution of the unconstrained analysis, no differences will arise in the estimates of the other parameters. A feasible way to achieve this, is to center the external configuration (if necessary) and center the data such that the corresponding configuration of the unconstrained solution is centered as well.

A similar problem exists with regard to possible rotations of the fixed $G$ and/or $H$ components. Because in TUCKALS2 and TUCKALS3 $G$ and $H$ are restricted to be columnwise orthonormal, it is necessary to orthonormalize the fixed $G$ and/or $H$ as well. The particular orientations of the axes of the fixed spaces are irrelevant for the fit, because rigid rotations of $G$ and $H$ are taken care of by corresponding transformations of the $C_{k}$.

TABLE 1

Centered and Orthonormalized Configurations of Fourteen Traits in Two Studies by Rosenberg

\begin{tabular}{|c|c|c|c|c|}
\hline \multirow[b]{2}{*}{ Trait } & \multicolumn{2}{|c|}{$\begin{array}{l}\text { Rosenberg } \\
\text { et al., } 1968\end{array}$} & \multicolumn{2}{|c|}{$\begin{array}{l}\text { Rosenberg } \varepsilon \\
\text { Sedlak, } 1972\end{array}$} \\
\hline & Dim. 1 & Dim. 2 & $\operatorname{Dim} .1$ & Dim. 2 \\
\hline Intelligent & .11 & .64 & .35 & .53 \\
\hline Honest & .18 & .14 & .31 & .04 \\
\hline Helpful & .23 & .04 & .17 & -.06 \\
\hline Sincere & .25 & -.01 & .27 & -.12 \\
\hline Humoristic & .19 & -.15 & .12 & -.22 \\
\hline Happy & .29 & -.13 & .05 & -.22 \\
\hline Good-natured & .26 & -.17 & -.33 & -.20 \\
\hline Warm & .28 & -.19 & .26 & -.31 \\
\hline Naive & -.18 & -.21 & -.01 & -.14 \\
\hline Unintelligent & -.33 & -.35 & -.38 & -.19 \\
\hline Irresponsible & -.38 & -.33 & -.29 & -.17 \\
\hline Moody & -.31 & .16 & .13 & .23 \\
\hline Cold & -.36 & .30 & -.19 & .42 \\
\hline Domineering & -.23 & .27 & -.46 & .39 \\
\hline
\end{tabular}

Note. These coordinates were derived from the figures presented in the Rosenberg and Sedlak paper. 
TABLE 2

Orthonormalized Configuration of Nine Attributes used by Rosenberg and Sedlak

\begin{tabular}{lrr}
\hline Attribute & Dim. 1 & Dim. 2 \\
\hline Intellectual good & .365 & -.151 \\
Social good & .301 & -.454 \\
Soft & .013 & -.551 \\
Passive & -.463 & -.085 \\
Impulsive & .362 & .155 \\
Bad & -.268 & .484 \\
Introvert & -.379 & -.199 \\
Dominant & .327 & .393 \\
Decided & .329 & .089 \\
\hline
\end{tabular}

\section{Example}

In order to illustrate external analysis with TUCKALS2 and TUCKALS3 we collected ratings of fourteen stimuli on nine attributes by seventeen subjects. External structures were available, both for the stimuli and the attributes, from two studies by Rosenberg and his colleagues (Rosenberg et al., 1968; Rosenberg \& Sedlak, 1972).

\section{Method}

Stimuli. The stimuli were the 14 personality trait adjectives that were included in both studies of Rosenberg. In these studies, dissimilarities between the traits were submit-

TABLE 3

Fit and Component Weights in

Ten External and Two Unconstrained TUCKALS Analyses

\begin{tabular}{lccccccc}
\hline \multicolumn{7}{c}{ component weights on two dimensions } \\
\cline { 2 - 6 } & \multicolumn{2}{c}{ stimuli } & attributes & \multicolumn{2}{c}{ subjects } & \\
analysis & 1 & 2 & 1 & 2 & 1 & 2 & total \\
\hline TUCKALS2: & & & & & & & \\
unconstrained & .407 & .155 & .401 & .162 & - & - & .562 \\
G68 fixed & .384 & .098 & .380 & .102 & - & - & .482 \\
G72 fixed & .173 & .124 & .211 & .085 & - & - & .297 \\
H72 fixed & .388 & .132 & .272 & .247 & - & - & .520 \\
G68 and H72 & .373 & .048 & .203 & .218 & - & - & .421 \\
G72 and H72 & .160 & .087 & .095 & .152 & - & - & .247 \\
TUCKALS3: & & & & & & & \\
unconstrained & .404 & .140 & .389 & .156 & .526 & .019 & .545 \\
G68 fixed & .383 & .084 & .372 & .095 & .453 & .015 & .467 \\
G72 fixed & .166 & .119 & .206 & .079 & .274 & .011 & .285 \\
H72 fixed & .385 & .117 & .262 & .240 & .483 & .018 & .502 \\
G68 and H72 & .372 & .038 & .197 & .213 & .391 & .019 & .410 \\
G72 and H72 & .158 & .080 & .089 & .148 & .226 & .011 & .237 \\
\hline
\end{tabular}

Note. Fit and component weights are expressed as proportions explained variation. 
ted to MDS, which resulted in two two-dimensional configurations of traits. The stimuli are listed in Table 1 together with their (centered and orthonormalized) coordinates.

Attributes. Ratings were obtained on nine attributes, the same attributes that were used by Rosenberg and Sedlak (1972) for interpreting the trait configuration. These attributes included the five properties that Rosenberg et al. (1968) had used for the same purpose. Table 2 contains a list of these attributes and their (orthonormalized) loadings on the first two principal components that were computed from the three varimax rotated factors presented in the 1972 paper.

Subjects. The subjects were 8 male and 9 female psychology and education students. They received a booklet consisting of an instruction followed by 14 pages, each containing one stimulus preceding the nine rating scales. The order of the stimuli, and the orders of the scales on each page were randomized. A final page contained questions about the subject's sex, age, and frequency of use of the stimulus and attribute words. The subjects completed the questionnaires individually at home or at the psychology department. They were rewarded with a copy of the Rosenberg and Sedlak paper (1972).

\section{Results}

Five external TUCKALS2, five external TUCKALS3 and two unconstrained analyses were run. In the external analyses, the fixed values for $G$ and $H$ were the stimulus coordinates of Rosenberg's 1968 and 1972 studies (G68 and G72, respectively), and the factor loadings of the attributes found in 1972 (H72). Since G72 and H72 were obtained from different samples of subjects, we also submitted combinations of G68 and $\mathbf{H} 72$ for

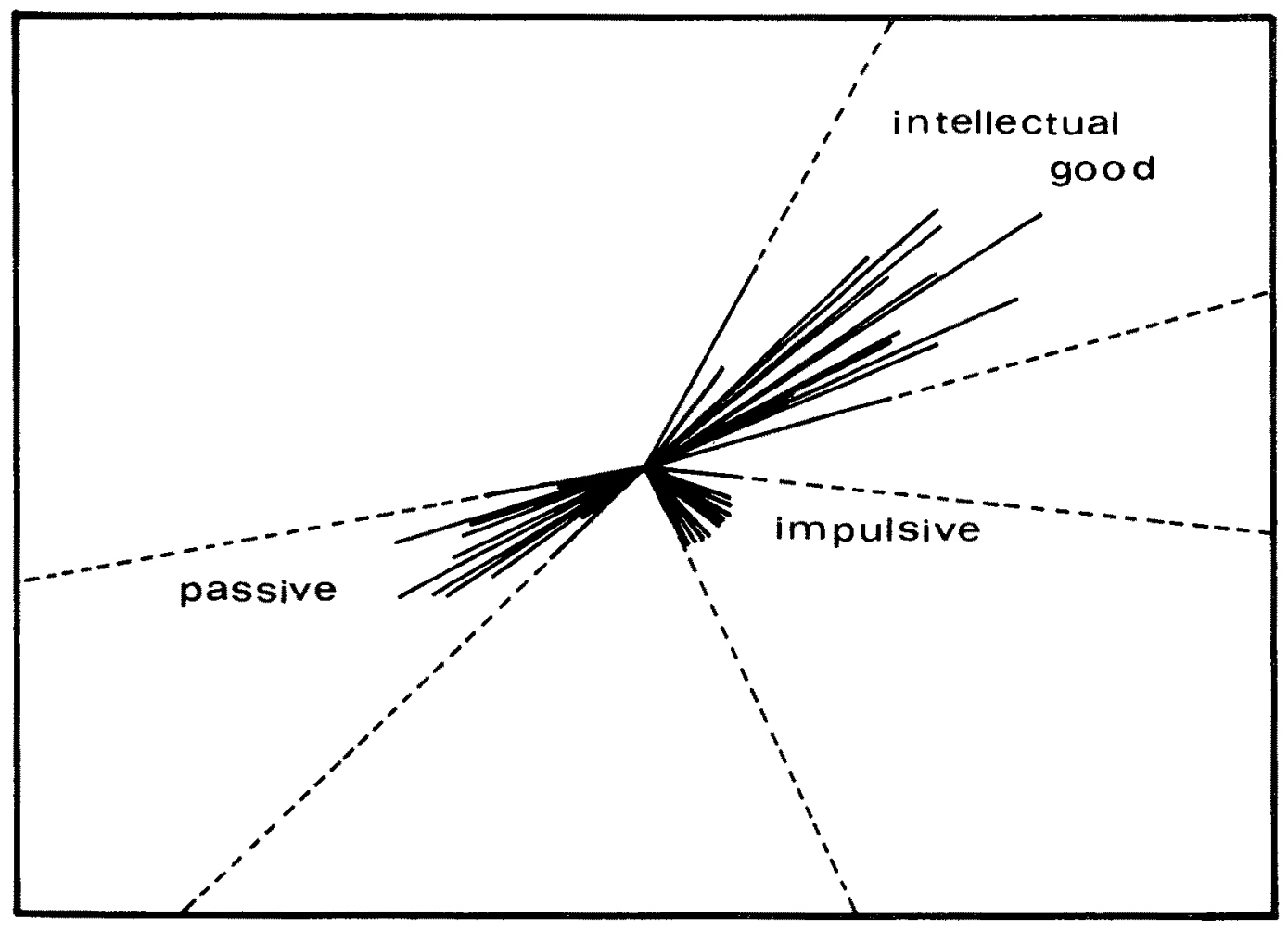

Figure 3

Individual vectors of the attributes intellectual good, passive, and impulsive in the G68 stimulus space. 


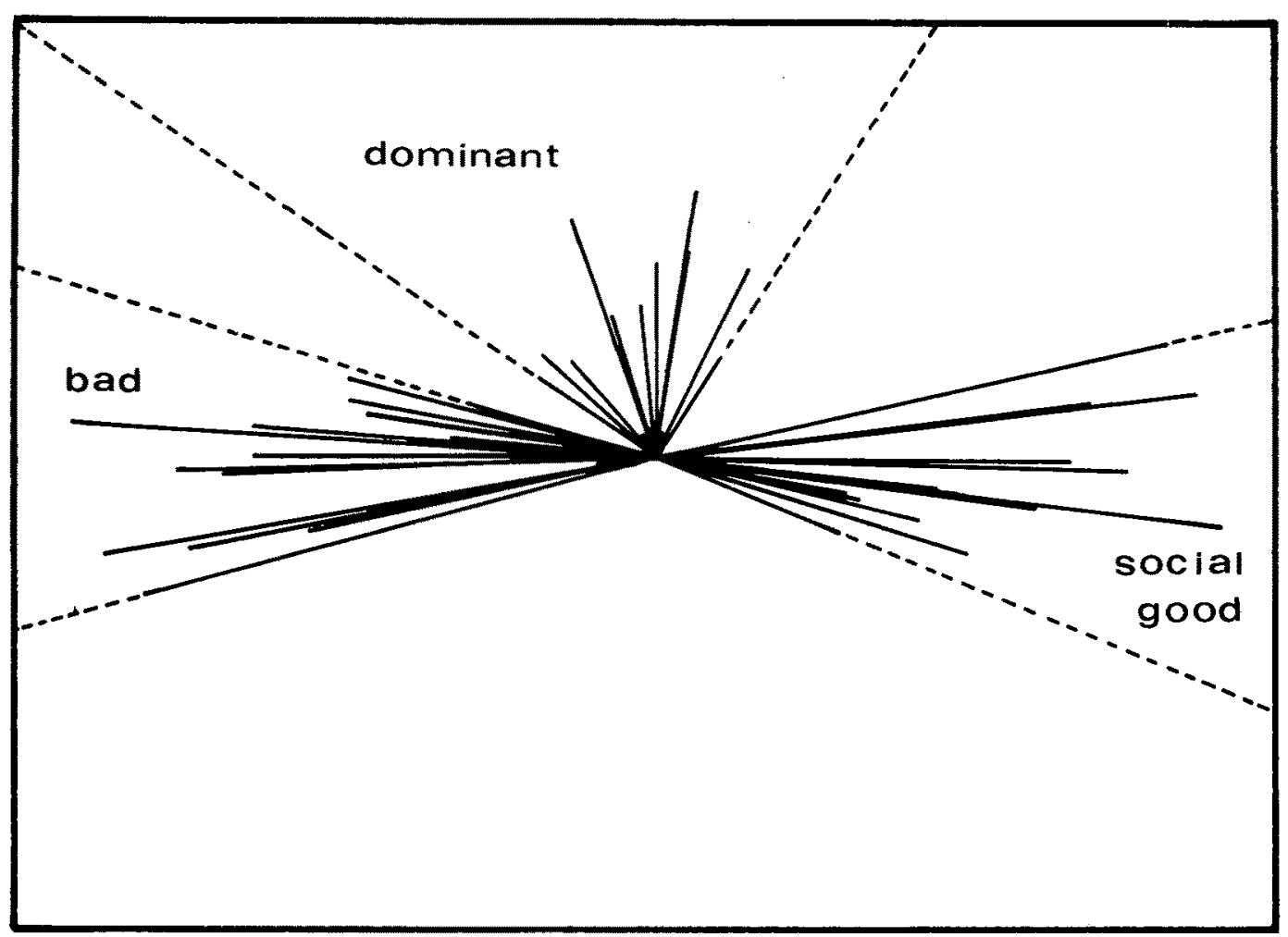

FIGURE 4

Individual vectors of the attributes social good, bad, and dominant in the G68 stimulus space.

external analysis. In all analyses both the stimulus and the attribute spaces were chosen to be two-dimensional. In the TUCKALS3 analyses the subjects were represented in two dimensions as well.

Measures of the fit and component weights of each analysis, expressed in terms of proportions explained variation, are given in Table 3. This table shows that there are six external analyses that approximate the fit of the corresponding unconstrained solutions. Those are the TUCKALS2 and TUCKALS3 analyses with G68 and/or H72 as fixed spaces. The fit values of the constrained solutions with $\mathrm{H} 72$ fixed are somewhat higher than those of the solutions with G68 fixed. This suggests that the loadings of the attributes are more stable or general than the loadings of the stimuli. Furthermore it is shown that there are only slight differences in fit between the TUCKALS2 and TUCKALS3 solutions, both in the external and in the unconstrained analyses. As the subject mode of the TUCKALS3 solutions appears to be essentially one-dimensional (i.e., the ratios of the component weights of the subject mode vary from $20: 1$ to $30: 1$ ) one may conclude that the $C_{k}$ matrices of the subjects are roughly proportional to each other. Therefore, the attribute ratings may be aggregated over the subjects.

In order to inspect the results graphically, we have plotted the attribute vectors for each individual into the G68 stimulus space using $H C_{k}^{\prime}$ as the coordinates (see Figure 3, 4 and 5). These figures show that the vectors for a particular attribute fall in a relatively narrow region. The largest variation is found for "dominant" for which the vectors of the individuals have a maximum angle of approximately 90 degrees. Figure 6, finally, shows the representation of the attributes in the stimulus space according to the first subject component from the TUCKALS3 analysis. The coordinates of the attributes were com- 


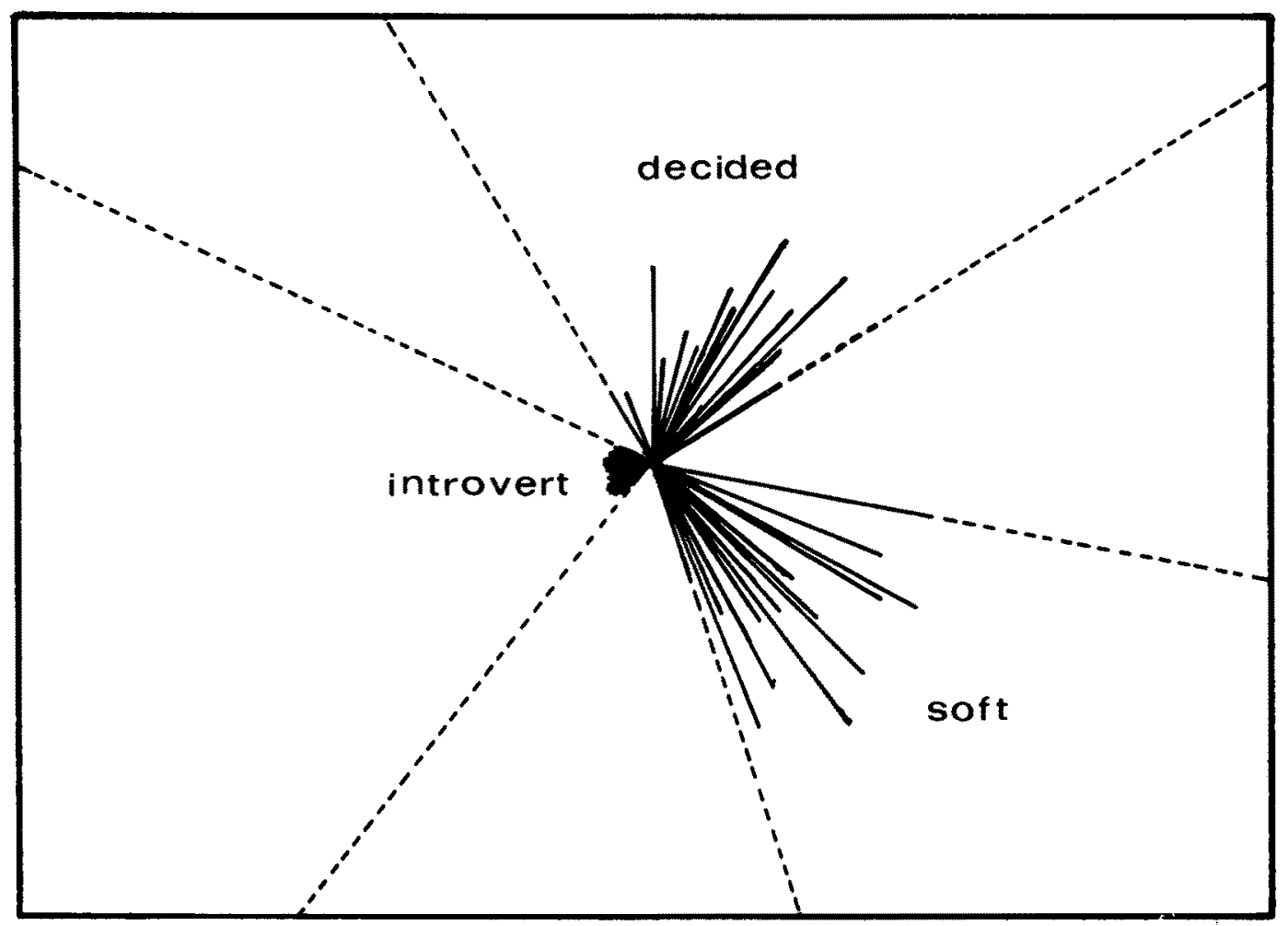

Figure 5

Individual vectors of the attributes soft, introvert, and decided in the G68 stimulus space.

puted as $\sum_{k=1}^{17}\left(e_{k 1} H C_{1}^{\prime}\right) / 17$, see (6). Superimposing this figure on Figures 3,4 and 5 would show that the TUCKALS3 attributes are roughly the mean directions of the bundles of individual vectors. These mean directions are very similar to the directions found by Rosenberg et al. in 1968. Also the amount of variation explained by each attribute in Rosenberg's and in our study, is the same order of magnitude.

Apart from being an illustration of external three-mode PCA, our example yielded some results that are of substantive interest. In the first place the results show that the outcomes of three independent studies, that differ in time, place, subjects, design, etcetera, are substantially similar. That is, the structure of the data in our example resembles the structure of the stimuli found by Rosenberg et al. (1968) and the structure of the attributes found by Rosenberg and Sedlak (1972). The core matrix of the unconstrained TUCKALS3 solution indicated that the axes of the attribute and the stimulus spaces practically coincide. Those axes can be interpreted as an evaluation and a dominance-submission dimension, and thus closely resemble the structures found by, for instance, Wiggins (1979) and van der Kloot and Kroonenberg (1982). In addition our analyses show that the variation among the subjects is relatively small.

\section{Discussion}

We have demonstrated that external analysis (or fix) options can be built into the TUCKALS 2 and TUCKALS3 algorithms, and that these techniques can be used to explore individual differences in the perception of stimuli in terms of a set of attributes. In particular, external three-mode PCA is useful for deciding whether the judgments of dif- 


\section{intel}

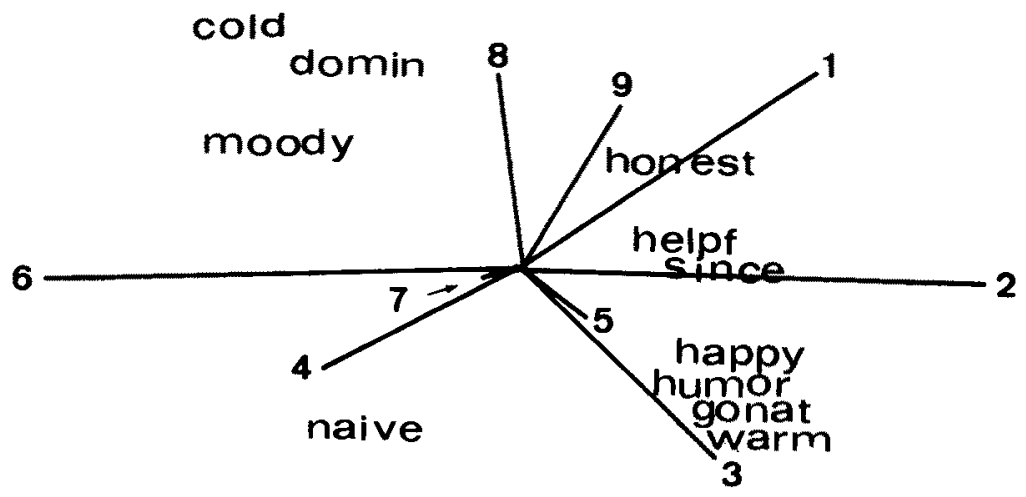

irres

unint

Figure 6

Attributes in the G68 stimulus space according to the first TUCKALS3 subject component. With the exception of honest and gonat (good-natured), the stimuli are labeled by the first five letters of their names (see Table 1). The fourteen stimuli are labeled by numbers corresponding to their order of appearance in Table 2.

ferent subjects may be aggregated or not. We have argued that aggregation is appropriate when different judges have attribute vectors that differ only in length and not in direction.

We have presented this "difference-in-length" versus "difference-in-direction" problem primarily within the framework of external analysis. The considerations presented are, however, equally valid in an unconstrained analysis in which one desires to represent the elements of one mode in the space of another mode that is not externally fixed.

Even though we have presented external three-mode PCA in terms of judges who rate a number of stimuli on a number of attributes, this approach is not limited to such data. Next to data with judges or subjects in the "replication mode," one may also apply external three-mode PCA to profile data (i.e., scores of subjects on variables) that are replicated in different situations or experimental conditions, or to multivariate longitudinal data with time as the replication mode.

There is a number of extensions possible for the methods we have described. In the first place, the orthonormality restrictions on the fixed space(s) may not always be realistic. To accommodate other than orthonormal fixed spaces, extensive adaptations of the algorithms are needed, the details of which have not been worked out yet. Secondly, it could be argued that comparisons between solutions should not only be based on proportions of fitted sums of squares, but should also involve the corresponding degrees of 
freedom. The question of the correct number of degrees of freedom in three-mode data, however, has not yet been solved completely (Kruskal, 1976, 1977, 1983b).

Because in external three-mode PCA a set of attributes is fitted into a fixed and untransformed stimulus space, it has been suggested (F. W. Young, personal communication, July 1983) that there might exist a relationship between our method and redundancy analysis (van den Wollenberg, 1977) and that it might be regarded as a threeway extension of the latter. This relationship, however, is not straightforward because different loss functions are minimized. As this issue is unrelated to the main thrust of this paper, it will be discussed elsewhere.

The substantive results of our example show that external three-mode PCA is a useful technique that enables one (a) to interpret stimulus spaces on the basis of attribute ratings by more than one judge, (b) to explore individual differences among those judges, and (c) to integrate the results of different and independently conducted studies. That external three-mode PCA does not always yield nice results is shown by Kroonenberg et al. (1983), who performed an external analysis on the data from a Cola tasting experiment by Schiffman et al. (1981).

${ }^{1}$ There also exists a French school dealing with three-mode or triadic PCA, but its work consists mainly of unpublished doctoral theses, and is not publicly available (e.g., Glacon, 1981; Jaffrenou, 1978; Mizère, 1981).

\section{References}

Bentler, P. M., \& Bonett, D. G. (1980). Significance tests and goodness of fit in the analysis of covariance structures. Psychological Bulletin, 88, 588-606.

Benzécri, J. P. (1976). L'Analyse des données. II. L'Analyse des correspondances [Data Analysis. II. Correspondence Analysis (2nd ed.)]. Paris: Dunod.

Benzécri, J. P., \& Benzécri, F. (1980). Pratique de l'analyse des données. 1. Analyse des correspondances. Exposé élémentaire [Practical Data Analysis. I. Correspondence Analysis. Introduction]. Paris: Dunod.

Bonett, D. G., \& Bentler, P. M. (1983). Goodness-of-fit procedures for the evaluation and selection of log-linear models. Psychological Bulletin, 93, 149-166.

Carroll, J. D. (1972). Individual differences and multidimensional scaling. In R. N. Shepard, A. K. Romney, \& S. B. Nerlove (Eds.), Multidimensional scaling: Theory and applications in the behavioral sciences, Vol. I: Theory (pp. 105-155). New York: Seminar Press.

Carroll, J. D., \& Chang, J. J. (1970). Analysis of individual differences in multidimensional scaling via an $N$-way generalization of "Eckart-Young" decomposition. Psychometrika, 35, 283-320.

Cazes, P. (1982). Note sur les éléments supplémentaires en analyse des correspondances. I. Pratique et utilisation. II. Tableaux multiples [Note on external variables in correspondence analysis. I. Utilization. II. Higher-way tables]. Les Cahiers de l'Analyse des Données, 7, 9-23, 133-154.

Glaçon, F. (1981). Analyse conjointe de plusieurs matrices de données [Conjoint analysis of several data matrices]. Unpublished doctoral dissertation, l'Université Scientifique et Médicinale de Grenoble, France.

Harshman, R. A. (1970). Foundations of the PARAFAC procedure. UCLA Working Papers in Phonetics, 16, 1-84. (University Microfilms International, Ann Arbor, MI, Order No. 10085)

Harshman, R. A. (1972). PARAFAC2: Mathematical and technical notes. UCLA Working Papers in Phonetics, 22, 30-44. (University Microfilms International, Ann Arbor, MI, Order No. 10085)

Harshman, R. A., \& Lundy, M. E. (1984a). The PARAFAC model for three-way factor analysis and multidimensional scaling. In H. G. Law, C. W. Snyder Jr., J. A. Hattie, \& R. P. McDonald (Eds.), Research methods for multi-mode data analysis (pp. 122-215). New York: Praeger.

Harshman, R. A., \& Lundy, M. E. (1984b). Data preprocessing and the extended PARAFAC model. In H. G. Law, C. W. Snyder, Jr., J. A. Hattie, \& R. P. McDonald (Eds.), Research methods for multi-mode data analysis (pp. 216-284). New York: Praeger.

Jaffrenou, P. A. (1978). Sur l'analyse des familles finies des variables vectorielles: Bases algébrique et application à la description statistique [On the analysis of finite sets of vector variables: Algebraic foundations and applications in descriptive statistics]. Unpublished doctoral dissertation, l'Université de Sainte- Etiene, France.

Kroonenberg, P. M. (1983). Three-mode principal component analysis: Theory and applications. Leiden: DSWO Press. 
Kroonenberg, P. M., \& de Leeuw, J. (1980). Principal component analysis of three-mode data by means of alternating least squares algorithms. Psychometrika, 45, 69-97.

Kroonenberg, P. M., van der Kloot, W. A., \& Brouwer, P. (1983, July). External three-mode principal component analysis. Paper presented at the 3rd European Meeting of the Psychometric Society, Jouy-en-Josas, France.

Kruskal, J. B. (1976). More factors than subjects, tests, and treatments: An indeterminacy theorem for canonical decomposition and individual differences scaling. Psychometrika, 41, 281-293.

Kruskal, J. B. (1977). Three-way arrays: Rank and uniqueness of trilinear decompositions with application to arithmetic complexity and statistics. Linear Algebra and its Applications, 18, 95-138.

Kruskal, J. B. (1981). Multilinear models for data-analysis. Behaviormetrika, 10, 1-20.

Kruskal, J. B. (1983a). Multilinear methods. In R. Gnadadesikan (Ed.), Statistical data analysis (pp. 36-62). Providence, RI: American Mathematical Society.

Kruskal, J. B. (1983b). Rank and geometry of three-dimensional matrices. Unpublished manuscript.

Mizère, D. (1981). Analyse d'une cube de données. Decomposition tensorielle et les liens entre procedures de comparaison de tableaux rectangulaires de données [Analysis of three-mode data. Tensor decomposition and relationships between procedures to compare rectangular data matrices]. Thèse de troisième cycle. l'Université Scientifique et Médicinale de Grenoble, France.

Ramsay, J. O. (1982). Multiscale II manual. Montreal, Canada: McGill University, Department of Psychology.

Rao, L. R. (1964). The use and interpretation of principal component analysis in applied research. Sankhya, 26(A), 329-358.

Rosenberg, S., Nelson, C., \& Vivekananthan, P. S. (1968). A multidimensional approach to the structure of personality impressions. Journal of Personality and Social Psychology, 9, 283-294.

Rosenberg, S., \& Sedlak, A. (1972). Structural representation of perceived personality trait relationships. In A. K. Romney, R. N. Shepard, \& S. B. Nerlove (Eds.), Multidimensional scaling: Theory and applications in the behavioral sciences, Vol. II: Applications (pp. 134-163). New York: Seminar Press.

Sands, R., \& Young, F. W. (1980). Component models for three-way data: ALSCOMP3, an alternating least squares algorithm with optimal scaling features. Psychometrika, 45, 39-67.

Schiffman, S. S., Reynolds, M. L., \& Young, F. W. (1981). Introduction to multidimensional scaling: Theory, methods, and applications. New York: Academic Press.

Tucker, L. R. (1966). Some mathematical notes on three-mode factor analysis. Psychometrika, 31. $279-311$.

Tucker, L. R. (1972). Relations between multidimensional scaling and three-mode factor analysis. Psychometrika, 37, 3-27.

van den Wollenberg, A. (1977). Redundancy analysis: An alternative for canonical correlation analysis. Psychometrika, 42, 207-219.

van der Kloot, W. A., \& Kroonenberg, P. M. (1982). Group and individual implicit theories of personality: An application of three-mode principal component analysis. Multivariate Behavioral Research, 17, 471-492.

Wiggins, J. S. (1979). A psychological theory of trait-descriptive terms: The interpersonal domain. Journal of Personality and Social Psychology, 37, 395-412.

Young, F. W., \& Lewyckyj, R. (1979). ALSCAL-4 user's guide. Carrboro, NC: Data Analysis and Theory Associates.

Manuscript received 6/30/83

Final version received $5 / 29 / 85$ 